\title{
An unusual Presentation of Dieulafoy's Lesion as a Cause of Recurrent Lower Gastrointestinal/Rectal Bleeding: A Case Report
}

\author{
Nazish Malik ${ }^{1, *}$, Munawwar Hussain ${ }^{1}$, Syed Mussadiq Ali Akbar Naqvi ${ }^{2}$ \\ ${ }^{1}$ Department of Internal Medicine, Conemaugh Memorial Medical Center, Johnstown PA, USA \\ ${ }^{2}$ Department of Internal Medicine, Parkland Hospital Dallas, TX, USA \\ *Corresponding author: dr.nazishmalik874@gmail.com
}

\begin{abstract}
Background: Dieulafoy lesion is a rare cause of acute gastrointestinal bleeding. It can present with sudden and massive bleeding without any prior symptoms. It causes massive hemorrhage leading to hemodynamic instability and this complicated nature of disease puts emphasis on its immediate diagnosis and treatment. Most commonly it presents with upper GIT bleeding but it can present with lower gastrointestinal bleeding. Case Presentation: We report a case of recurrent lower gastrointestinal bleeding that was miss diagnosed initially as internal hemorrhoids. A 91-year-old male was discharged 2 weeks ago, who presented again with rectal bleeding and low hemoglobin found on lab reports. He was admitted and transfused two packs of PRBC. After not finding any visible bleeding on upper endoscopy, he had colonoscopy and a bleeding vessel was found in rectum. Clipping was performed to stop bleeding and he was discharged home. Lower gastrointestinal bleeding is a very rare presentation of dieulafoy lesion. Conclusion: Dieulafoy lesion can present with upper or lower gastrointestinal bleeding. Diagnostic improvements and research for the detection of DL needs to be conducted to reduce hospital stay and improve survival. Endoscopic measures, mechanical banding, hemoclipping and electrocoagulation are some of the frequently used treatment options.
\end{abstract}

Keywords: Dieulafoy's lesion, recurrent bleeding, internal hemorrhoids, diagnostic approaches

Cite This Article: Nazish Malik, Munawwar Hussain, and Syed Mussadiq Ali Akbar Naqvi, "An unusual Presentation of Dieulafoy's Lesion as a Cause of Recurrent Lower Gastrointestinal/Rectal Bleeding: A Case Report." American Journal of Medical Case Reports, vol. 5, no. 10 (2017): 259-261. doi: 10.12691/ajmcr-5-10-2.

\section{Background/ Introduction}

Obscure gastrointestinal bleeding can occur in 5\% of cases and Dieulafoy's lesion is one of the rare causes [1]. It is also known as dieulafoy ulcer or constant diameter artery bleeding, which is a rare as well as under-recognized cause of bleeding arteries. It is always considered in differential diagnosis of obscure gastrointestinal bleeding because of its uncertain and serious nature it should be diagnosed and managed promptly. It can't be identified by conventional methods like endoscopy or barium studies and is often misdiagnosed as hemorrhoids. Normally, an artery narrows down progressively as it passes through wall of its end organ. In Dieulafoy's lesion artery maintains a constant diameter and becomes tortuous in submucosa [1]. Most commonly it occurs in proximal stomach but less commonly can involve rectum, colon and small intestine.

\section{Case Presentation}

A 91-year-old male patient presented with fresh bleeding per rectum, weakness and low hemoglobin as lab finding. Two weeks ago, he was diagnosed with internal hemorrhoids when he presented with lower GI bleed. He had EGD done on that admission which reported superficial non-bleeding gastric ulcers. He had colonoscopy and diagnosed with internal hemorrhoids. He was stabilized and discharged home initially. Now he presented again with bleeding per rectum and abnormal labs. He had history of peptic ulcer disease, HTN, CKD stage 5, atrial fibrillation, chronic diastolic congestive heart failure and sick sinus syndrome status post pacemaker. He had no allergies, no significant family history, and a life time nonsmoker. He was taking atorvastatin, metoprolol, aspirin, eliquis and pantoprazole. He was vitally stable. His labs were normal except hemoglobin $7.8 \mathrm{gm} / \mathrm{dl}$, hematocrit $24 \%$, BUN 50 and creatinine $6.2 \mathrm{mg} / \mathrm{dl}$. He was given IV fluids and a nephrology consult was requested. On previous visit his hemoglobin was $10.5 \mathrm{gm} / \mathrm{dl}$ and his creatine level was $3.9 \mathrm{mg} / \mathrm{dl}$. Patient reported bright red rectal bleeding started one day ago. He was transfused 2 units of PRBC and gastroenterology consult was requested. Due to poor kidney function lisinopril and lasix were held. Aspirin and eliquis were held for ongoing bleeding. On second day of admission patients had endoscopy that didn't show any acute bleeding. On third day, he underwent colonoscopy. On colonoscopy blood was found in the rectum a bleeding 
rectal Dielafoy's lesion (Submucosal artery) was noted. Complete hemostasis was achieved with the deployment of two resolution clips. No further bleeding was noted and patient was discharged home in stable condition.
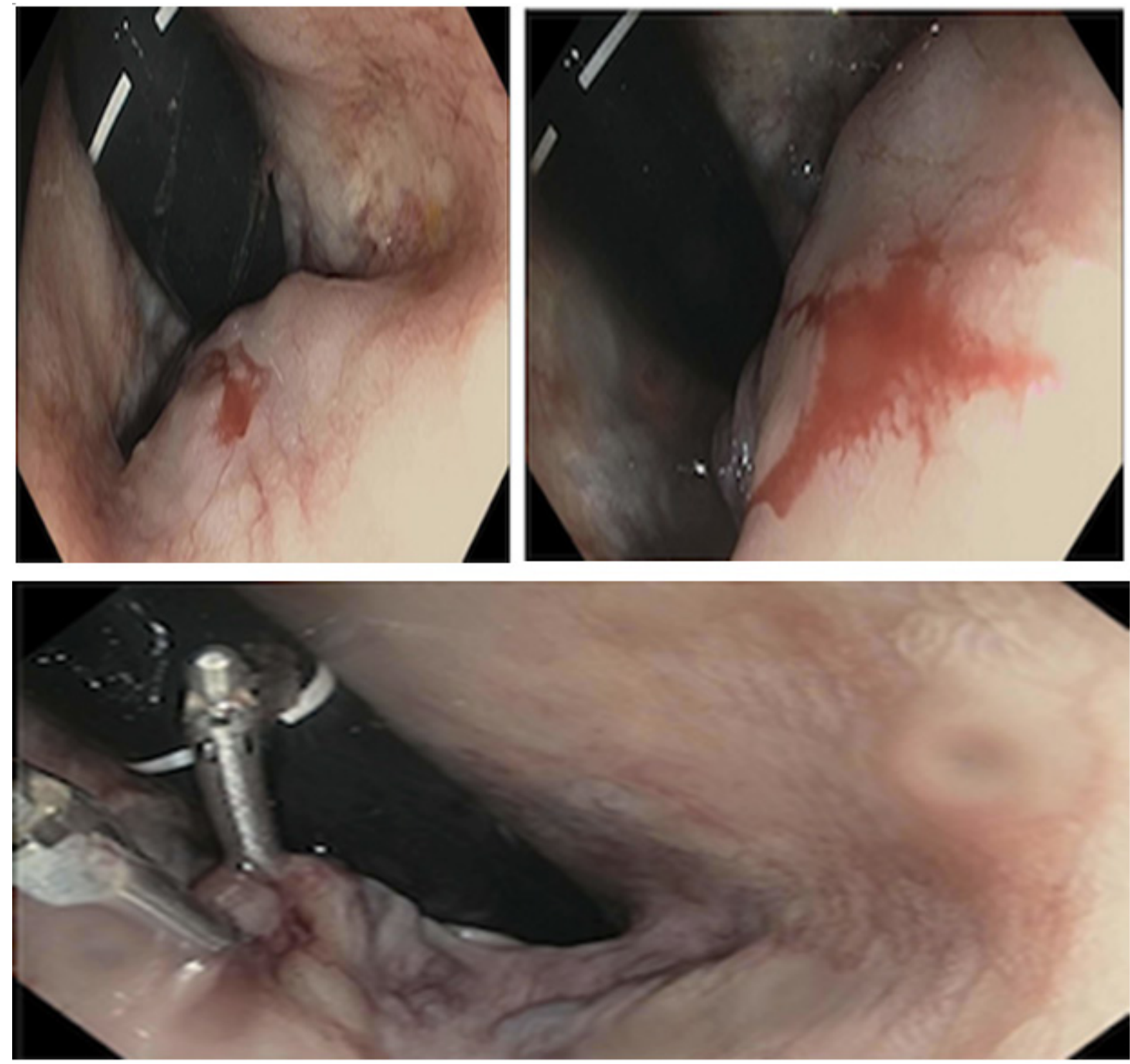

Figure 1.

\section{Case Discussion}

Dieulafoy lesion is twice as common in males as females, it can occur at any age. But most commonly presents in elderly population. It is evident from case histories that most of the patients presenting with bleeding are already hospitalized. Almost $90 \%$ of patients already have associated comorbidities like cardiopulmonary complications and chronic renal failure. Several mechanisms have been proposed to discuss the pathophysiology of DL. One theory suggests that pulsations in the vessel leads to disruption of the underlying epithelium which leads to rupture and subsequent massive hemorrhage [1]. Colonic DL presents with profuse bright red blood per rectum. Typically, bleeding is severe and acute due to large arterial vessels and arterial nature of bleeding [4]. Initial GI endoscopy is effective in diagnosing up to $70 \%$ of the cases [1]. The endoscopic criteria to define DL is active arterial spurting or micro pulsatile streaming from minute ( $<3 \mathrm{~mm}$ ) mucosal defect or through normal surrounding mucosa, visualization of a protruding vessel with or without active bleeding through defected or normal surrounding mucosa and fresh densely adherent clot with a narrow point of attachment to defected or normal surrounding mucosa [3]. It is very difficult to diagnose DL and should be considered in every patient presenting with recurrent, massive and acute GI bleeding. In recent years, more cases are diagnosed on first endoscopy, in some cases it remains undiagnosed till repeat endoscopy or until angiography is done. DL can be easily missed as awareness of pathology and careful endoscopy are key to accurate diagnosis [2]. Colonoscopy is usually indicated when endoscopy fails to find a cause of acute GI bleeding. Multiple cases of DL diagnosed on colonoscopy has been reported in past 30 years [4].

Therapeutic endoscopy is the procedure of choice for initial treatment of DL. Adrenaline injection is also used frequently with or without endoscopic procedures. Other endoscopic techniques include laser photocoagulation, injection sclerotherapy, bipolar and monopolar electrocoagulation, endoscopic band ligation and hemoclipping. Surgical ligation is used in less than 
5\% case where endoscopy fails. Lastly, angiography and embolization are considered in patients where endoscopy and surgical approaches both fail [2]. We used hemoclipping for this patient. The key points for successful clipping are as follows: (1) the lesion needs to be directly observed; (2) the lesion and its surrounding tissue should be fully exposed, and the angle of the clip and bleeding site should be in a range of $45-90^{\circ}$; and (3) the depth of the clip should be considered. The optimal depth is where the exposed vessel and deep tissue can be clipped. The clip should not be superficial, and if it is superficial, the clip can come off in a short period, and then re-bleeding is inevitable [5].

\section{Conclusion}

DL mostly occurs in upper gastrointestinal tract and presents with profuse acute hemorrhage, but in rare cases it can occur in lower GI where it presents with fresh bleeding per rectum. Careful diagnostic and therapeutic approaches should be used in evaluating patients presenting with GI bleeding. Great attention should be paid to make correct diagnosis, as it can be easily overlooked or misdiagnosed. That can be life threatening [5].

\section{Abbreviations}

DL; Dieulafoy, lesion;

GIT: Gastrointestinal tract

EGD: Esophagogastroduodenoscopy

PRBC packed red blood cells.

\section{References}

[1] Baxter, M., \& Aly, E. (2010). Dieulafoy's lesion: current trends in diagnosis and management. Annals of The Royal College of Surgeons of England, 92(7), 548-554.

[2] Ibrarullah, M., \& Wagholikar, G. D. (2003). Dieulafoy's lesion of duodenum: a case report. BMC Gastroenterology, 3, 2 .

[3] Apiratpracha, W., Ho, J. K., Powell, J. J., \& Yoshida, E. M. (2006). Acute lower gastrointestinal bleeding from a dieulafoy lesion proximal to the anorectal junction post-orthotopic liver transplant. World Journal of Gastroenterology: WJG, 12(46), 7547-7548.

[4] Nojkov, B., \& Cappell, M. S. (2015). Gastrointestinal bleeding from Dieulafoy's lesion: Clinical presentation, endoscopic findings, and endoscopic therapy. World Journal of Gastrointestinal Endoscopy, 7(4), 295-307.

[5] Cui, J., Huang, L.-Y., Liu, Y.-X., Song, B., Yi, L.-Z., Xu, N., ... Wu, C.-R. (2011). Efficacy of endoscopic therapy for gastrointestinal bleeding from Dieulafoy's lesion. World Journal of Gastroenterology : WJG, 17(10), 1368-1372. 